\title{
Influence of Circadian Typology and Time of Day on Temporal Preparation
}

\author{
Ángel Correa ${ }^{1,2}$, Tania Lara ${ }^{2}$ and Juan Antonio Madrid ${ }^{3}$ \\ 1. Departamento de Psicología Experimental, Universidad de Granada, Spain. \\ 2. Centro de Investigación Mente, Cerebro y Comportamiento, Universidad de Granada, Spain. \\ 3. Laboratorio de Cronobiología, Departamento de Fisiología, Universidad de Murcia, Spain.
}

SHORT TITLE: Circadian Rhythms and Temporal Preparation

NOTICE: This is the authors' version of a work that was accepted for publication, and can be used for scholarly non-commercial purposes. Changes resulting from the publishing process, such as editing, corrections, structural formatting, and other quality control mechanisms may not be reflected in this document. Definitive version will be subsequently published by the journal.

Corresponding author: Ángel Correa. Departamento de Psicología Experimental. Facultad de Psicología, Campus Universitario de Cartuja s/n, 18071-Granada, Spain. http://www.ugr.es/ act/. Email: act@ugr.es. Phone: +34 958 246240. Fax: +34 958246239. 


\begin{abstract}
The present study addressed the interactions between processes of circadian and millisecond timing by testing whether the ability for temporal preparation is influenced, both by individual differences in circadian rhythmicity and by the time of day at which a task is performed. A temporal preparation task that measures temporal orienting and sequential effects was administered to morning-type and evening-type groups of participants, both in morning and evening sessions. The results confirmed a synchrony effect on overall reaction time (RT), indicating that participants were most vigilant at their optimal time of day according to their specific chronotype. This synchrony effect, however, did not influence temporal orienting or sequential effects. These findings suggest that only processes mediating overall RT (vigilance) but not processes related to temporal preparation are susceptible to circadian influence. The current research then supported the dissociation between circadian timing and temporal preparation.
\end{abstract}

Keywords: attention, timing, go no-go, foreperiod, sequential effects, temporal orienting, chronotype, synchrony effect. 


\section{Introduction}

A fundamental biological function concerns the ability of timing natural events and regulate behaviour accordingly, at scales ranging from milliseconds to hours along the day (for reviews see Agostino, Golombek, \& Meck, 2011; Buhusi \& Meck, 2005). The current study investigated the influence of circadian timing on cognitive functions related to the temporal anticipation and preparation of responses to forthcoming events in the milliseconds to seconds range (i.e., 'temporal preparation'). We specifically tested whether temporal preparation would be most effective at specific times of day according to individual differences in circadian rhythmicity as indexed by the chronotype.

Most physiological and behavioural functions essential for healthy living (e.g., fluctuations of body temperature, awake-sleep or rest-activity states) follow a cycle of about 24 hours, known as circadian rhythm (Berendes et al., 1960; Kleitman, 1933). Research in Chronobiology has provided a variety of physiological markers of circadian rhythms, such as melatonin concentration, motor activity or body temperature. In humans, core body temperature (CBT) and peripheral skin temperature (PST) usually follows circadian rhythmicity with inverse daily patterns, respectively. While CBT fluctuates between minimums at night to rising values along the day, PST exhibits the opposite pattern (e.g., Baehr, Revelle, \& Eastman, 2000; Ortiz-Tudela, Martinez-Nicolas, Campos, Rol, \& Madrid, 2010; Sarabia, Rol, Mendiola, \& Madrid, 2008). Similarly, speed and accuracy during the performance of cognitive tasks, such as working memory, response inhibition or simple reaction time (RT) tasks, are also subject to circadian variations (reviewed by Blatter \& Cajochen, 2007; García, Ramírez, Martínez, \& Valdez, 2012; Valdez, Ramírez, García, Talamantes, \& Cortez, 2010; Wright, Hull, \& Czeisler, 2002). 
'Circadian typology' or 'chronotype' reflects different temporal profiles in the functioning of the circadian clock (recently reviewed by Adan et al., 2012; Horne \& Ostberg, 1976). Morning-type people prefer to wake up and to go to bed earlier than Evening-type people. These subjective preferences are also reflected objectively in circadian phases differing by 2-4 hours in most physiological variables (Baehr et al., 2000) and have a genetic basis (Katzenberg et al., 1998). Importantly, the same objective time of day can result optimal for some persons or especially inappropriate for others, depending on their chronotype. Thus, speed and accuracy during the performance of attention and memory tasks can be optimal in the morning or evening depending on the chronotype. This interaction between the influence of chronotype and time of day is known as the 'synchrony effect' (May \& Hasher, 1998).

May and Hasher (1998) found that performance in executive-control tasks indexing frontal functioning (stop-signal, stroop and trail making test B) was highest in the morning for morningtype (older) adults, and in the evening for evening-type (younger) participants. Executive control can be measured by tasks that demand the ability to select the appropriate response while inhibiting other distracting items (e.g., the go no-go task used in our current study). In May and Hasher's study, circadian variations were mainly observed on difficult conditions demanding controlled processing, but not on conditions of well-learned responses involving automatic processing (see also Manly, Lewis, Robertson, Watson, \& Datta, 2002; May, Hasher, \& Foong, 2005; West, Murphy, Armilio, Craik, \& Stuss, 2002).

An interesting research question raised by the above studies is to identify which cognitive processes are susceptible of the synchrony effect. For example, some studies suggest that the synchrony effect may involve higher level of alertness and vigilance at optimal vs. suboptimal times of day (Lim \& Dinges, 2008; Matchock \& Mordkoff, 2009). It might then be possible that these differential 
alertness states would influence the ability for temporal preparation, one of the basic cognitive functions related to alertness and timing. The current study tested this issue by comparing the behavioural performance during a temporal preparation task, which was administered at optimal vs. suboptimal times of day according to participants' chronotype.

Temporal preparation involves the ability to synchronise the optimal level of readiness to specific time intervals (in the range of milliseconds to seconds) at which a relevant event is expected to occur, in order to produce efficient performance. Behavioural advantages of temporal preparation can be measured in the laboratory through a variable foreperiod paradigm (reviewed by Correa, 2010). That is, a warning signal is presented, followed by a preparatory interval (so called 'foreperiod'), which duration (e.g., a few seconds) can change trial-by-trial, and the time to respond to a target stimulus is measured. In these conditions, RTs are typically faster for targets occurring at the long than at the short foreperiod, which has been termed 'the foreperiod effect' (reviewed by Niemi \& Näätänen, 1981).

Although temporal preparation is transient, its time course can be voluntarily adjusted to make the optimal state of preparation coincide with the moment at which a target is expected to occur ('temporal orienting of attention'; Coull \& Nobre, 1998). Temporal orienting has been studied by cuing individuals with explicit and predictive temporal information about the foreperiod duration. This information is the basis of temporal expectations about when the target is going to appear (e.g., early -after 400 ms or late -after 1400 ms) (reviews in Coull \& Nobre, 2008; Nobre, 2001). The temporal orienting effect refers to the relative performance benefit for responding to targets appearing at validly cued vs invalidly cued foreperiods. This validity effect is mainly found at the short foreperiod (faster RT for "early" cue - short foreperiod vs "late" cue - short foreperiod condition). Unless catch trials (trials where no target is presented) are used, the target ends up 
appearing with full probability at the long foreperiod, which reduces the effect of the temporal cue (Correa, Lupiáñez, Milliken, \& Tudela, 2004).

Temporal orienting has been related to processes of executive control. Neuroimaging research (Coull, Davranche, Nazarian, \& Vidal, 2012; Coull, Frith, Buchel, \& Nobre, 2000) consistently shows activation of fronto-parietal structures related to attentional control in the effect of temporal orienting. Neuropsychological studies further indicate that temporal orienting requires a functional right prefrontal cortex (Triviño, Arnedo, Lupiáñez, Chirivella, \& Correa, 2011; Triviño, Correa, Arnedo, \& Lupiáñez, 2010). Moreover, temporal orienting is impaired by the concurrent performance of a working memory secondary task (Capizzi, Correa, \& Sanabria, 2013; Capizzi, Sanabria, \& Correa, 2012). These studies further suggest that 'sequential effects' of temporal preparation, in contrast to temporal orienting, are independent of neural and cognitive processes related to executive control (Vallesi, Shallice, \& Walsh, 2007; Vallesi et al., 2007a; see also Steinborn \& Langner, 2011). In fact, sequential effects are considered to reflect automatic processing (Capizzi et al., 2012).

Sequential effects refer to the influence of the foreperiod duration of the previous trial upon the RT in the current trial. Specifically, RTs to a current short foreperiod are typically faster when the previous foreperiod was short rather than long. Sequential effects are usually asymmetric as the effect of the previous foreperiod is small or absent at the current long foreperiod. The same explanation based on conditional probability (i.e., the foreperiod effect) as applied for temporal orienting, can be applied here for the asymmetry of sequential effects (but see Los \& Van den Heuvel, 2001, for a different account). Therefore, temporal preparation is not a unitary process (Nobre, Correa, \& Coull, 2007). 
In the current study we used a task that measures both temporal orienting and sequential effects, to test for the first time (to the best of our knowledge), whether time of day and individual differences in circadian rhythmicity could influence temporal preparation. Our prediction relied on the two following findings: 1) the synchrony effect is observed on executive control rather than on automatic processes, and 2) temporal orienting, but not sequential effects, involves processes and neural networks related to executive control. Therefore, we predicted that only temporal orienting, but not sequential effects (automatic temporal preparation), would be influenced by the synchrony effect of circadian rhythms. That is, we expected to find an interaction between time of day, chronotype and validity of temporal cue, such that participants performing the temporal preparation task at their optimal time of day according to their chronotype would show larger temporal orienting effects as compared to performance at their non-optimal time of day. In contrast, sequential effects should not be affected by the synchrony effect.

\section{Materials and Methods}

\section{Participants}

The chronotype of 580 Psychology students from the University of Granada was measured by administering the reduced scale of the Morningness-Eveningness questionnaire (rMEQ; Adan \& Almirall, 1991). From this pool, we contacted potential participants with extreme scores in the rMEQ continuum and 32 of them accepted to participate in this study in exchange of course credits. Data from 5 participants who had slept less than 5 hours the night prior to any of the two sessions were discarded, and data from 2 participants could not be analysed because they missed the second experimental session. The final sample then consisted of 10 morning-type (mean age: 23 years, range: $19-31$, SD: 3.7; 7 females) and 15 evening-type participants (mean age: 23 years, range: 18- 
33, SD: $3.9 ; 10$ females). The two experiments were conducted according to the ethical standards of the Universidad de Granada and in accordance to the 2008 Declaration of Helsinki.

\section{Apparatus and stimulus}

The chronotype was measured by the reduced scale of the Morningness-Eveningness questionnaire (Adan \& Almirall, 1991), which was adapted from Horne and Östberg (1976) and standardised for the Spanish population. Scores can range between 4 (extreme eveningness) and 25 (extreme morningness). Participants' body temperature was measured at the armpit with a digital thermometer. Subjetive activation and subjective affect were measured by visual analogue scales (Monk, 1989). Scores can range from 0 (minimum either activation or positive mood, respectively) to 100 (maximum activation or positive mood). Temporal preparation was measured by a behavioural RT task that was designed and administered through E-prime software (Schneider, Eschman, \& Zuccolotto, 2002). The task stimuli and procedure had been used in previous research (for further details see Correa, Miró, Martínez, Sánchez, \& Lupiáñez, 2011; Correa, Triviño, PérezDueñas, Acosta, \& Lupiáñez, 2010, the task can be downloaded here: http:/www.ugr.es/ act/paper/10Correa_B\&C_Task.es.zip). All the stimuli were presented at the centre of the computer screen over a black background. The temporal cue was either a short bar $\left(0.38^{\circ} \times 0.95^{\circ}\right)$ or a long bar $\left(0.38^{\circ} \times 2.1^{\circ}\right)$. The short bar indicated that the target would appear early (after $400 \mathrm{~ms}$ ), and the long one that the target would appear late (after $1400 \mathrm{~ms}$ ). The go target $\left(0.38^{\circ} \times 0.76^{\circ}\right)$ was either the letter ' $\mathrm{O}$ ', or the letter ' $\mathrm{X}$ ', whereas the no-go target was the digit ' 8 '. There were $25 \%$ of trials that included the no-go target. In the go condition, participants pressed the ' $\mathrm{B}$ ' key whenever an ' $\mathrm{O}$ ' or an ' $\mathrm{X}$ ' appeared. In the no-go condition, participants should inhibit responding. 


\section{Procedure}

The participants completed the same experimental protocol twice. The experimental sessions were scheduled at two different times of day, and delivered in two different days separated by a week. Morning and evening sessions were scheduled at 10 am and $9 \mathrm{pm}$, respectively. The order of sessions was counterbalanced across participants, with half of the morning group starting the first session in the morning and the remaining half in the evening. Similarly, approximately half of the evening group ( 7 out of 15 ) completed the first session in the morning.

When the participants arrived at the laboratory, they were seated in a room under dim light conditions $(<8$ lux $)$ and after a few minutes their body temperature was measured by placing a thermometer in their armpit for about 5 minutes. In the meantime, they responded several questions regarding sleeping (amount of sleeping the night before the experiment, waking time) and use of stimulant drinks, and then they fulfilled the visual analogue scale of activation and affect. Then, participants were placed at a viewing distance of about $60 \mathrm{~cm}$ and performed the temporal preparation go no-go task. They were instructed to respond as quickly as possible only to the go targets and to avoid responding to the no-go target. Each trial began with the fixation point presented for a random duration between 500 and $1500 \mathrm{~ms}$, followed by the temporal cue (short or long bar presented in red for $50 \mathrm{~ms}$ ). Next, the screen remained blank for a variable delay of 350 or $1350 \mathrm{~ms}$ depending on the foreperiod for that trial. The target was displayed for $100 \mathrm{~ms}$ and was then replaced by a blank screen until the participant made a response or for a maximum duration of $2000 \mathrm{~ms}$.

The experiment included one practice block and 4 experimental blocks. The experimental blocks were divided into 2 'early' blocks, in which the short bar indicated that the target would probably 
appear after $400 \mathrm{~ms}$, and 2 'late' blocks, in which the long bar indicated that the target would probably appear after $1400 \mathrm{~ms}$ (cue validity: 75\%). Thus, temporal expectancy was manipulated between blocks to optimise temporal orienting effects (Correa, Sanabria, Spence, Tudela, \& Lupiáñez, 2006). 'Early' and 'late' blocks were presented in alternating runs, and the order of presentation was counterbalanced across participants. Each experimental block included 32 trials that were randomly presented on a trial-by-trial basis $(25 \%$ were no-go trials, and foreperiods were distributed to have $75 \%$ of valid cues).

\section{Design and data analysis}

Duration of sleeping the night prior the experiment, body temperature, subjective activation, subjective affect and behavioural performance in the temporal preparation task (RTs and errors of failed inhibition) were submitted to separate analyses of variance (ANOVA) with the following main design: Chronotype (morning-type, evening-type) as between participants factor, and Time of Day (morning, evening) manipulated within participants. Moreover, we tested for potential differences between morning-type and evening-type groups in terms of both chronotype score in the rMEQ and chronological age.

In the behavioural task, practice trials, the first trial of each block and trials with anticipatory responses before target onset (51 anticipations only) were not analysed. The RT analysis computed mean RTs between 200 and $800 \mathrm{~ms}(0.85 \%$ of trials rejected $)$ from correct responses. The error analysis computed mean error percentages from responses in the no-go condition. Both RTs and errors were submitted to separate ANOVAs. As in previous research using this temporal preparation task (Correa et al., 2010), temporal orienting effects were measured by an ANOVA between Validity of the temporal cue (valid, invalid) and Foreperiod (short, long), whereas sequential effects were 
measured by an ANOVA between duration of Previous foreperiod (short, long) and Current Foreperiod (short, long), where the two levels of the Validity factor were collapsed. In order to test whether temporal orienting and sequential effects were influenced by circadian factors, these analyses included chronotype and time of day. Power analyses were performed by using the noncentral F distribution (Steiger \& Fouladi, 1997) and a value of 0.3 as root mean square standardized effect (medium effect according to Cohen, 1983).

\section{Results}

\section{Demographic data}

One participant reported to drink coffee within the 4 hours prior to the session at her/his nonoptimal time of day. The ANOVA of the rMEQ scores with Chronotype (morning-type, eveningtype) as between participants factor confirmed that the morning-type group scored significantly higher in morningness (M: 18.4, SD: 0.97) than the evening-type group (M: 8.8, SD: 2.04), $F(1,23)$ $=190.39, p<.001$. The analysis of age confirmed that the two groups were matched in this variable, $F<1$. The ANOVA of sleeping duration in the night prior the experiment showed that the morning-type group slept significantly longer (7.9 hours) than the evening-type group (6.8 hours), $F(1,23)=10.34, p<.01$. Importantly, this difference was not associated with time of day (chronotype $\mathrm{x}$ time of day: $F<1$ ), so that the amount of sleeping on the night before was similar for both sessions.

Body temperature and subjective state data

Body temperature ${ }^{1}$ was higher in the evening $\left(36.65^{\circ} \mathrm{C}\right)$ than in the morning session $\left(36.37^{\circ} \mathrm{C}\right)$, $F(1,19)=5.93, p=.02$, although this effect did not interact with chronotype $(F<1)$. The ANOVAs 
of subjective activation and affect did not show significant effects in this experiment.

\section{Behavioural data}

Table 1 represents the mean RTs and SD of responses to the go condition, and percentage of errors of failed inhibition in the no-go condition, for each experimental condition in Experiment 1.

Table 1. Mean RTs, SD [between brackets] and percentage of errors in the no-go condition (in parenthesis) from Experiment 1 broken down by chronotype (morning-type, evening-type) and time of day (morning, evening) for temporal orienting (validity $\mathrm{x}$ foreperiod) and sequential effects (previous foreperiod x current foreperiod).

Temporal Orienting

Sequential Effects

\begin{tabular}{llll}
\hline Short Foreperiod & Long Foreperiod & Previous Short & Previous Long
\end{tabular}

Valid Invalid Valid Invalid Short $\quad$ Long $\quad$ Short $\quad$ Long

\begin{tabular}{lccccccccc}
\hline Morning-Type & RT & 423 & 450 & 424 & 422 & 421 & 444 & 423 & 425 \\
Morning session & {$[\mathrm{SD}]$} & {$[49]$} & {$[54]$} & {$[33]$} & {$[44]$} & {$[48]$} & {$[54]$} & {$[37]$} & {$[34]$} \\
& $(\%$ error $)$ & $(12)$ & $(2)$ & $(16)$ & $(10)$ & $(15)$ & $(14)$ & $(7)$ & $(11)$ \\
\hline Morning-Type & $\mathrm{RT}$ & 443 & 463 & 447 & 451 & 443 & 454 & 446 & 449 \\
Evening session & {$[\mathrm{SD}]$} & {$[57]$} & {$[56]$} & {$[37]$} & {$[52]$} & {$[59]$} & {$[54]$} & {$[40]$} & {$[42]$} \\
& $(\%$ error $)$ & $(17)$ & $(12)$ & $(14)$ & $(8)$ & $(13)$ & $(12)$ & $(14)$ & $(18)$ \\
\hline Evening-Type & $\mathrm{RT}$ & 412 & 437 & 424 & 428 & 406 & 438 & 423 & 426 \\
Morning session & {$[\mathrm{SD}]$} & {$[27]$} & {$[40]$} & {$[21]$} & {$[25]$} & {$[27]$} & {$[35]$} & {$[24]$} & {$[20]$} \\
& $(\%$ error $)$ & $(17)$ & $(9)$ & $(17)$ & $(20)$ & $(21)$ & $(13)$ & $(11)$ & $(17)$ \\
\hline Evening-Type & $\mathrm{RT}$ & 394 & 413 & 407 & 410 & 386 & 416 & 407 & 408 \\
Evening session & {$[\mathrm{SD}]$} & {$[35]$} & {$[34]$} & {$[38]$} & {$[46]$} & {$[31]$} & {$[40]$} & {$[43]$} & {$[39]$} \\
\hline
\end{tabular}


In the temporal orienting RT analysis, the ANOVA between chronotype, time of day, cue validity and foreperiod showed a significant interaction between chronotype and time of day, $F(1,23)=$ $7.24, p=.01$. Figure 1 shows the typical synchrony effect, as time of day produced opposite effects on overall RTs depending on chronotype. That is, each chronotype group responded fastest when the cognitive task was performed at its optimal time of day. Planned comparisons revealed that evening-type participants tended to respond faster in the evening than in the morning session, $F(1,23)=4.12, p=.054$, whereas morning-type participants tended to respond faster in the morning than in the evening, $F(1,23)=3.3, p=.08$.

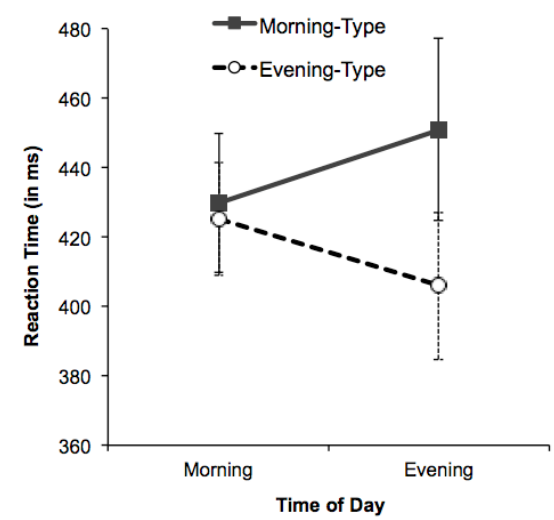

Figure 1. Mean reaction times as a function of time of day (morning $-10 \mathrm{am}$, evening $-9 \mathrm{pm}$ ) in morning-type and evening-type groups in Experiment 1. Vertical bars represent standard errors of the mean.

Moreover, the results replicated the usual findings with this temporal preparation task, namely, a significant main effect of validity (i.e., the 'temporal orienting effect'), $F(1,23)=27.47, p<.001$, with faster RTs on valid (422 ms) than on invalid (434 ms) trials, and a significant interaction between validity and foreperiod, $F(1,23)=24.06, p<.001$, which showed that the validity effect 
was present only at the short foreperiod, $F(1,23)=34.69, p<.001$, but not at the long foreperiod $(F<1)$. However, as can be observed in Fig. 2, the pattern of validity effects did not change according to time of day or chronotype (chronotype $\mathrm{x}$ time of day $\mathrm{x}$ validity $\mathrm{x}$ foreperiod: $F<1$; chronotype $\mathrm{x}$ time of day $\mathrm{x}$ validity: $F<1$ ). Further analysis of the chronotype $\mathrm{x}$ time of day $\mathrm{x}$ validity design yielded a statistical power of 0.847 . The remaining main effects and interactions were not significant either (all $p s>.18$, except for a marginally significant effect of chronotype, $F(1,23)=3.64, p=.07$, with evening-type responding faster than morning-type). The error analysis showed a main effect of validity, $F(1,23)=8.64, p<.01$, with more errors of failed response inhibition in valid $(16 \%)$ than in invalid trials $(11 \%)$.
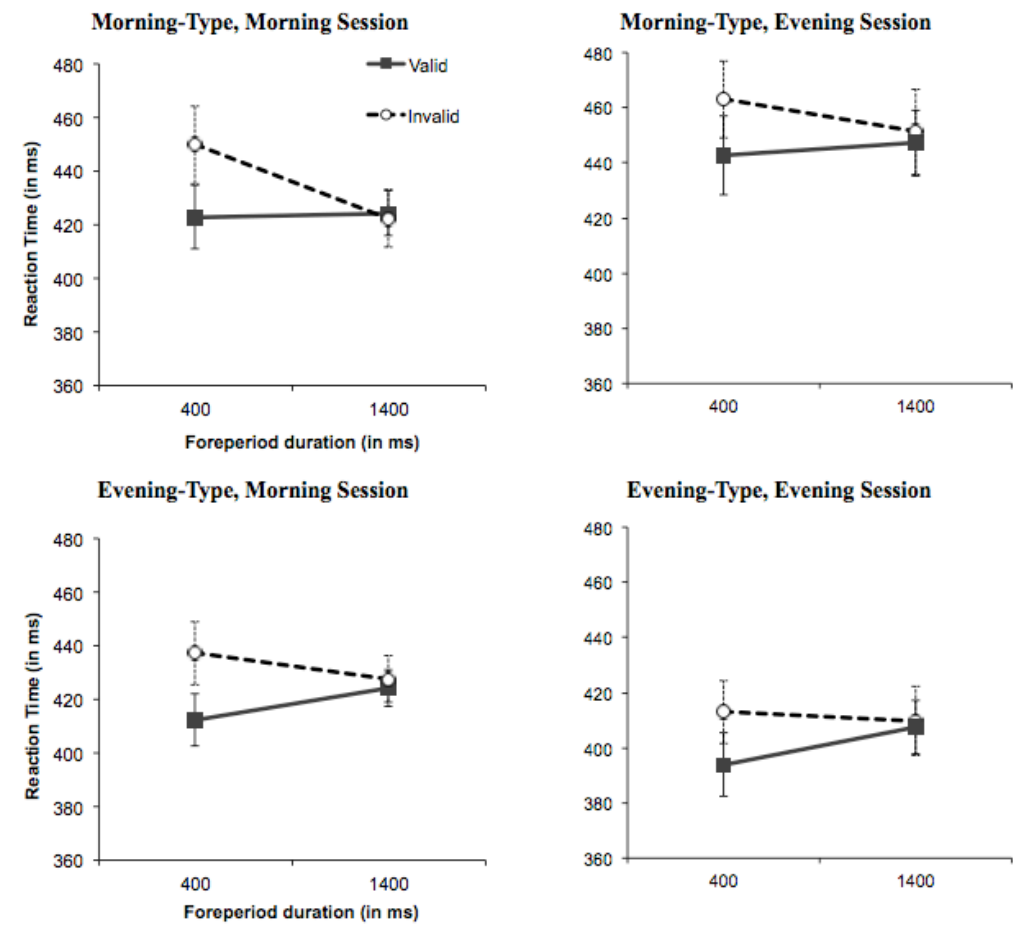

Figure 2. Mean reaction times (in ms) as a function of cue validity (valid: solid line, invalid: dashed line), foreperiod duration in the X-axis (short $-400 \mathrm{~ms}$, long $-1400 \mathrm{~ms}$ ) and time of day (morning: left panels, evening: right panels) in morning-type (top panels) and evening-type (bottom panels) groups in Experiment 1. Vertical bars represent standard errors of the mean. Temporal orienting (i.e., the validity effect at the short foreperiod) was significant in all four conditions, but was not influenced by circadian factors (chronotype x time of day x validity: $F<1$ ). 
In the sequential effects RT analysis, the ANOVA of chronotype, time of day, foreperiod and previous foreperiod revealed a significant main effect of previous foreperiod, $F(1,23)=38.17, p<$ .001 , with faster RTs when the previous foreperiod was short (419 ms) rather than long (433 ms). The typical asymmetry of this sequential effect was confirmed by the significant interaction between previous foreperiod and current foreperiod, $F(1,23)=20.53, p<.001$, since the effect of the previous foreperiod was only significant at the current short foreperiod, $F(1,23)=38.44, p<$ .001 , but not at the current long foreperiod $(F<1)$. As can be observed in Fig. 3, the pattern of sequential effects did not change according to time of day or chronotype (chronotype $\mathrm{x}$ time of day $\mathrm{x}$ foreperiod $\mathrm{x}$ previous foreperiod: $F<1$ ). The remaining main effects or interactions did not approach significance (all ps >.1).
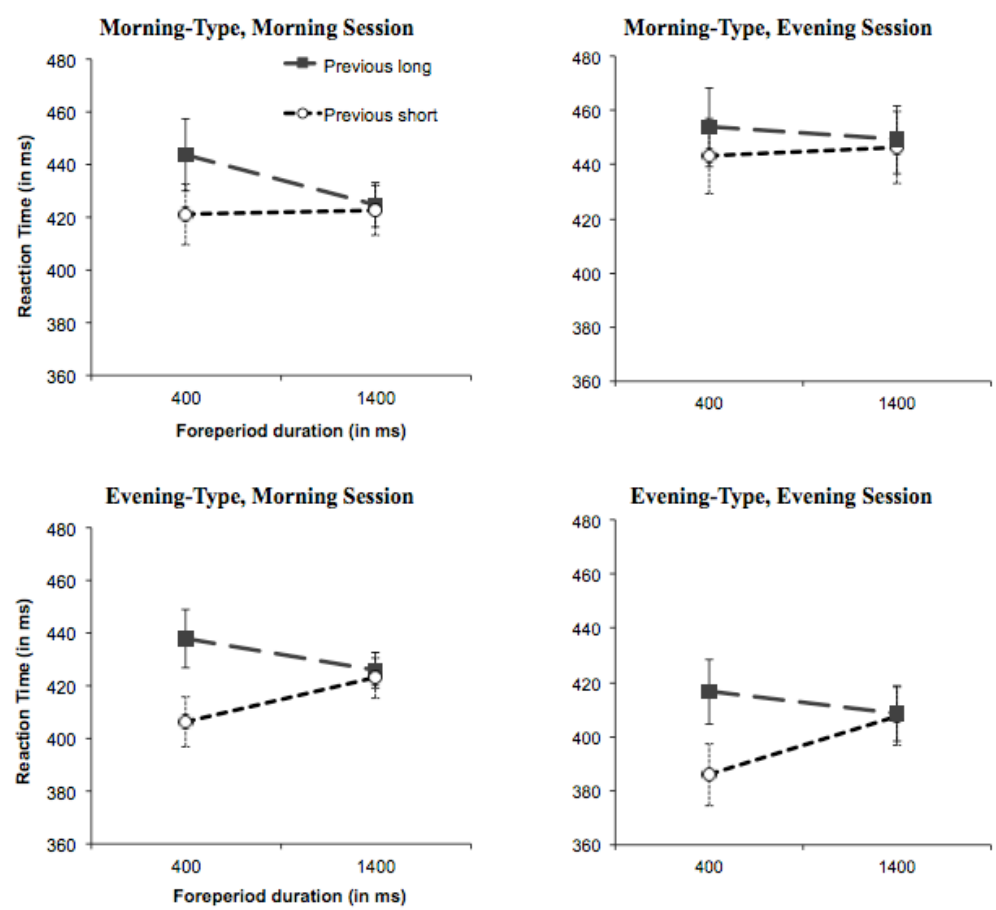

Figure 3. Mean reaction times as a function of previous foreperiod (short: shorter dashed line, long: longer dashed line), current foreperiod in the x-axis (short $-400 \mathrm{~ms}$, long - $1400 \mathrm{~ms}$ ), and time of day (morning, evening) in morning-type and evening-type groups in Experiment 1. Vertical bars represent standard errors of the mean. Sequential effects were significant (previous foreperiod $\mathrm{x}$ 
current foreperiod, $F(1,23)=20.53, p<.001)$, but they did not change according to time of day or chronotype (chronotype $\mathrm{x}$ time of day $\mathrm{x}$ previous foreperiod $\mathrm{x}$ current foreperiod: $F<1$ ).

The error analysis showed a significant interaction between previous and current foreperiod, $F(1,23)=20.17, p<.001$, showing more errors when previous and current foreperiods were of similar duration.

\section{Discussion}

Experiment 1 tested whether two effects of temporal preparation (temporal orienting and sequential effects) were influenced both by the time of day when the task is performed and by individual differences in circadian rhythmicity (i.e., chronotype). According to research showing that the synchrony effect (i.e., interaction between time of day and chronotype) influence selectively cognitive functions that require executive control rather than automatic processing (Manly et al., 2002; Matchock \& Mordkoff, 2009; May et al., 2005), and given that temporal orienting involves controlled processing while sequential effects involve automatic processing (Capizzi, et al., 2012), we expected to find that temporal orienting but not sequential effects were modulated by circadian factors. The finding of circadian effects on body temperature and overall RT in the behavioural task confirmed the effectiveness of our manipulation of both chronotype and time of day. However, the results did not show any circadian influence on temporal orienting. Sequential effects, as expected, were not affected by the circadian manipulation either.

The finding of higher armpit temperature in the evening as compared to the morning session corresponded to the normal daily pattern of CBT, and supported that participants actually were in two different circadian phases (Baehr et al., 2000). However, the sampling of only two points along 
the circadian cycle was insufficient to capture different trends across chronotypes in our experiment. Most relevant, we found a synchrony effect on the overall RT in the behavioural task. Confirming our hypothesis, the fastest responses were observed when participants performed the task at their optimal time of day according to their chronotype. This finding suggests that morningtype participants had higher levels of vigilance in the morning than in the evening session, while evening-type participants tended to be more vigilant in the evening than in the morning (see Langner, Steinborn, Chatterjee, Sturm, \& Willmes, 2010, for a similar argument). The critical question here was whether this differential level of vigilance covaried with the ability of temporal preparation.

The replication of typical validity and sequential effects confirmed that the temporal preparation task generally worked as expected (Correa et al., 2004). As an exception, we found that validity effects produced faster responses, but at the cost of increasing errors of failed inhibition in the nogo condition. This speed-accuracy trade off has been described for the effects of phasic alertness induced by warning signals (Posner, 1978), and is congruent with the fact that warning signals, similarly to temporal cues, usually provide reliable temporal information that can be used to predict forthcoming stimulus onset. This result is also congruent with the finding that temporal orienting can interfere with response selection in flanker and Simon tasks (Correa, Cappucci, Nobre, \& Lupiáñez, 2010), since the current go no-go task similarly involved executive control for response selection and inhibition. Sequential effects showed a similar speed-accuracy trade off, such that conditions of higher preparation (short-short sequence) led to more inhibition errors than conditions of less preparation (long-short sequence).

On the other hand, both effects of temporal preparation resisted the influence of chronotype and time of day. This resistance was expected for sequential effects but not for temporal orienting. 
However, it could be argued that the current procedure, in which temporal cues were manipulated between blocks rather than within blocks, did not really measure controlled aspects of temporal orienting. Indeed, recent evidence supports this argument: Capizzi et al. (2012) showed that temporal orienting was impaired by the concurrent performance of a working memory task, which competes for cognitive resources of executive control. However, this interference effect was found only when the temporal cue was manipulated within-block on a trial-by-trial basis, but not when it was manipulated between blocks, as in the current experiment. Capizzi et al. therefore concluded that temporal orienting in a between-blocks design and sequential effects both involve automatic processes of temporal preparation that do not require executive control, whereas temporal orienting in a within-block design involves controlled temporal preparation.

So far, the interim conclusion of Experiment 1 would be that chronotype and time of day did not influence automatic aspects of temporal preparation, such as those involved in temporal orienting in a between-blocks design and sequential effects. The aim of Experiment 2 was to test whether these circadian factors would influence controlled temporal orienting, that is, when cues were manipulated on a trial-by-trial basis.

\section{Experiment 2}

Experiment 2 tested the influence of chronotype and time of day on temporal preparation when the cue inducing temporal expectancy could change from trial to trial, rather than between different blocks (Experiment 1). Given that controlled processing depends on circadian factors, we expected that controlled temporal orienting but not automatic sequential effects would show the synchrony effect as revealed by the interaction between chronotype and time of day. 


\section{Materials and methods}

Everything was similar to Experiment 1 except for the following changes.

\section{Participants}

Participants with extreme scores in the rMEQ were contacted from the same pool of Experiment 1 and 44 of them accepted to participate in this study. Data from 8 participants who had slept less than 5 hours the night prior to one session were discarded, data from 2 participants were discarded due to $0 \%$ accuracy in the no-go condition, and data from 4 participants could not be analysed because they missed the second experimental session. The final sample consisted of 14 morning-type (mean age: 22 years, range: 18-31, SD: 4; 6 males) and 16 evening-type participants (mean age: 21 years, range: $18-27$, SD: $2.9 ; 7$ males).

\section{Design and data analysis}

In the behavioural task, practice trials, the first trial of each block and trials with anticipatory responses before target onset (only 67 anticipations) were not analysed. The RT analysis computed mean RTs between 200 and $800 \mathrm{~ms}$ ( $0.8 \%$ of trials rejected) from correct responses.

\section{Results}

Demographic data

Seven participants (4 evening-type and 3 morning-type) drank coffee within the 4 hours prior to the 
session at their non-optimal time of day. Six of them also drank coffee at their optimal time of day session. Therefore, the effects of caffeine were well balanced for both optimal and nonoptimal times of day (Ryan, Hatfield, \& Hofstetter, 2002). The ANOVA of the rMEQ scores, with chronotype (morning-type, evening-type) as between groups factor, confirmed that the morning-type group scored significantly higher in morningness (M: 17.9, SD: 1.17) than the evening-type group (M: 9.7, SD: 2.9), $F(1,28)=220.96, p<.001$. Also, the groups were matched in terms of age, $F<1$. The ANOVA of the sleeping duration in the night previous to the experimental session confirmed that the two groups were balanced in the amount of sleeping (main effect of chronotype: $F<1$, chronotype x time of day: $F<1)$.

\section{Body temperature and subjective state data}

Replicating Experiment 1, temperature was higher in the evening $\left(36.35^{\circ} \mathrm{C}\right)$ than in the morning session $\left(36.07^{\circ} \mathrm{C}\right), F(1,25)=6.39, p=.02$, although this effect did not interact with chronotype $(F$ $<1)$. The ANOVA of subjective activation replicated the synchrony effect by showing a significant interaction between chronotype and time of day, $F(1,27)=21.26, p<.001$. Planned comparisons indicated that morning-type participants reported to be more alert in the morning than in the evening session, $F(1,27)=9.37, p<.01$, whereas evening-type participants felt most alert in the evening than in the morning session, $F(1,27)=12.19, p<.01$. The ANOVA of subjective affect did not show any significant effects (all Fs $<1$ ).

\section{Behavioural data}

Table 2 represents the mean RTs and SD, and percentage of errors of failed inhibition for each experimental condition in Experiment 2. 
Table 2. Mean RTs, SD [between brackets] and percentage of errors in the no-go condition (in parenthesis) from Experiment 2 broken down by chronotype (morning-type, evening-type) and time of day (morning, evening) for temporal orienting (validity $\mathrm{x}$ foreperiod) and sequential effects (previous foreperiod $\mathrm{x}$ current foreperiod).

Temporal Orienting

Sequential Effects

Short Foreperiod

Long Foreperiod

Previous Short

Previous Long

\begin{tabular}{|c|c|c|c|c|c|c|c|c|c|}
\hline & & & & & & & & & \\
\hline & & Valid & Invalid & Valid & Invalid & Short & Long & Short & Long \\
\hline Morning-Tyne & RT & 414 & 410 & 422 & 418 & 406 & 421 & 417 & 425 \\
\hline & {$[\mathrm{SD}]$} & [33] & [37] & [31] & [37] & [35] & [33] & [32] & [32] \\
\hline & (\% error) & $(20)$ & (21) & (22) & (16) & (23) & $(16)$ & (19) & (21) \\
\hline Mornino-Tyne & RT & 419 & 427 & 430 & 427 & 411 & 432 & 430 & 427 \\
\hline & {$[\mathrm{SD}]$} & [32] & [33] & [39] & [40] & [33] & [32] & [38] & [40] \\
\hline ש & ( $\%$ error) & $(25)$ & (14) & $(20)$ & (23) & (24) & $(20)$ & $(17)$ & (22) \\
\hline Fyening_Tyne & RT & 421 & 441 & 431 & 438 & 421 & 432 & 431 & 435 \\
\hline & {$[\mathrm{SD}]$} & [32] & [36] & [36] & [44] & [34] & [32] & [39] & [37] \\
\hline 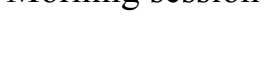 & (\% error) & $(12)$ & (13) & $(12)$ & (9) & (12) & $(11)$ & (9) & (13) \\
\hline Evening-Type & RT & 404 & 422 & 414 & 421 & 400 & 417 & 414 & 418 \\
\hline & {$[\mathrm{SD}]$} & [41] & [34] & [42] & [48] & [36] & [43] & [42] & [44] \\
\hline & (\% error) & (18) & (12) & (15) & (18) & (20) & (13) & (13) & (18) \\
\hline
\end{tabular}

In the temporal orienting RT analysis ${ }^{2}$, the ANOVA between chronotype, time of day, cue validity and foreperiod replicated the interaction found in Experiment 1 between chronotype and time of day on the overall RT, $F(1,28)=4.18, p=.05$. Planned comparisons showed that evening-type participants tended to respond faster in the evening than in the morning session, $F(1,28)=3.75, p=$ 
.06. The effect of time of day was not significant for morning-type participants $(F<1)$.

The main effect of cue validity was significant, $F(1,28)=6.83, p=.01$, with faster RTs on valid (419 ms) than on invalid (425 ms) trials. As in Experiment 1, this effect was significant at the short foreperiod (validity effect of $10 \mathrm{~ms}, F(1,28)=8.18, p<.01$ ) but not at the long one $(F<1)$. A significant interaction between validity and chronotype, $F(1,28)=9.03, p<.01$, showed validity effects in evening-type (validity effect of $13 \mathrm{~ms}, F(1,28)=16.92, p<.001$ ) but not in morning-type $(F<1)$ participants. More relevant for our main hypothesis, and replicating Experiment 1, the pattern of validity effects did not change as a function of time of day (chronotype $\mathrm{x}$ time of day $\mathrm{x}$ validity $\mathrm{x}$ foreperiod: $F<1$; time of day $\mathrm{x}$ validity $\mathrm{x}$ foreperiod: $F<1$ ). The analysis of the chronotype $\mathrm{x}$ time of day $\mathrm{x}$ validity design yielded a statistical power of 0.905 .

The error analysis showed a significant interaction between time of day, validity and foreperiod, $F(1,28)=10.69, p<.01$, showing that validity effects at the short foreperiod were only significant in the evening session $(\mathrm{p}<.01)$ but not in the morning session $(F<1)$, whereas validity effects at the long foreperiod were not significant in either session (both $p s>.13$ )

In the sequential effects RT analysis, the ANOVA of chronotype, time of day, foreperiod and previous foreperiod revealed significant main effects of previous foreperiod, $F(1,28)=36.45, p<$ .001 , with faster RTs when the previous foreperiod was short $(416 \mathrm{~ms})$ rather than long (426 ms), and a main effect of foreperiod, $F(1,28)=4.82, p=.04$, with faster RTs at current short (417 ms) rather than long $(425 \mathrm{~ms}$ ) foreperiods. Sequential effects were asymmetric (previous foreperiod $\mathrm{x}$ current foreperiod: $F(1,28)=11.28, p<.01)$, since the effect of the previous foreperiod was significant at the current short foreperiod only, $F(1,28)=44.03, p<.001$, but not at the current long foreperiod $(\mathrm{p}>.24)$. Replicating Experiment 1, the pattern of sequential effects was not influenced 
by the synchrony effect (chronotype $\mathrm{x}$ time of day $\mathrm{x}$ foreperiod $\mathrm{x}$ previous foreperiod: $F<1$ ). The remaining main effects and interactions were far from significance (all $F s<1$ ), except for a marginally significant interaction between time of day, previous foreperiod and current foreperiod, $F(1,28)=3.01, p=.09$, such that sequential effects were symmetric in the morning but asymmetric in the evening session (previous foreperiod $\mathrm{x}$ current foreperiod, respectively: $F(1,28)=2.16, p=$ .15 and $F(1,28)=13.23, p<.01)$.

The error analysis only showed a significant interaction between previous and current foreperiod, $F(1,28)=4.61, p=.04$, which replicated Experiment 1 by showing more errors when previous and current foreperiods were of similar duration.

Further analyses were optimised to search for potential synchrony effects on temporal preparation (see Langner et al., 2010, for a similar approach). However, the analyses confirmed the main results of standard analyses, that is, no synchrony effects on either validity or sequential effects. Specifically, the RT analysis focused in the group of participants showing the largest synchrony effect (RT_non-optimal minus RT_optimal time of day), divided by median split. In the temporal orienting analysis, the time of testing (optimal vs. non-optimal) $\mathrm{x}$ validity (valid vs. invalid) $\mathrm{x}$ foreperiod (short vs. long) ANOVA confirmed that validity did not depend on time of testing (time of testing x validity $\mathrm{x}$ foreperiod: $F<1$; time of testing $\mathrm{x}$ validity: $F<1$ ). In the sequential effects analysis, the time of testing (optimal vs. non-optimal) $\mathrm{x}$ previous foreperiod (short vs. long) $\mathrm{x}$ foreperiod (short vs. long) ANOVA showed that effects of previous foreperiod did not depend on time of testing (time of testing x previous foreperiod: $F<1$; time of testing $\mathrm{x}$ previous foreperiod $\mathrm{x}$ foreperiod: $p>.25)$.

\section{Discussion}


Experiment 2 aimed to optimize the finding of circadian influences on the temporal orienting of attention by emphasizing the demands of controlled processing, that is, by manipulating temporal expectancy following a within-block design rather than the between-blocks manipulation followed in Experiment 1. However, the main pattern of results of Experiment 1 was replicated. The synchrony effect, as revealed by the interaction between chronotype and time of day, was significant for both self-reported activation and overall RT in the cognitive task, respectively, which warranted the effectiveness of our circadian manipulation. Nevertheless, this synchrony effect was not found in either temporal orienting or sequential effects.

The RT data showed an interaction between validity and chronotype, and the error data showed interaction between validity (at the short foreperiod) and time of day. Moreover, sequential effects on RT were clearly asymmetric only in the evening session. According to Vallesi and colleagues (e.g., Vallesi \& Shallice, 2007), the asymmetry of sequential effects is due to the influence of the foreperiod effect. That is, the effect of the previous foreperiod is reduced or eliminated at the current long foreperiod, where temporal expectancy about target onset is maximal (i.e., the conditional probability of target occurrence is 1 at the long foreperiod). Following this model, the monitoring of conditional probabilities requires the right dorsolateral prefrontal cortex, a neural structure involved in cognitive control. When this structure is not fully functional, for example as a result of either virtual lesions with transcranial magnetic stimulation (TMS) or insufficient brain development (in young children of only 4-5 years old), the monitoring of conditional probabilities is impaired, typically leading to symmetric sequential effects (Vallesi, et al., 2007b; Vallesi \& Shallice, 2007; Vallesi, et al., 2007a).

Our results fit with this explanation, such that temporal orienting as indexed by validity effects, and 
the foreperiod effect leading to asymmetric sequential effects, were more effective in the evening than in the morning. These results suggest that circadian factors can exert some influence on controlled aspects of temporal preparation. However, as mentioned above, this influence did not confirm our main hypothesis that validity effects would be larger at optimal vs. suboptimal times of day according to the participants' chronotype.

\section{General discussion}

This work aimed to investigate the relationship between two timing mechanisms operating at different time scales. The circadian clock regulates physiological and behavioural functions, preparing the organism for events unfolding with circadian rhythmicity within a period of $24 \mathrm{~h}$. On the other hand, temporal preparation can be developed in anticipation of events unfolding in the range of milliseconds to seconds. Previous studies have reported that circadian rhythms can affect time perception (reviewed by Agostino et al., 2011). However, it was unknown whether circadian rhythms may also determine temporal preparation, the ability to use temporal information to respond efficiently.

We conducted two experiments to test whether time of day and individual differences in circadian rhythmicity (chronotype) influence the controlled process of temporal preparation, as measured by a temporal orienting task in which expectancy was manipulated on a trial-by-trial basis (Experiment 2). In contrast, we expected automatic temporal preparation not to be affected by circadian factors. The results of the two experiments converged in showing a synchrony effect in overall RT of the temporal preparation task, which confirmed the effectiveness of our manipulation of both time of day and chronotype (see also Note 2). Furthermore, the temporal preparation task showed clear effects of temporal orienting and sequential foreperiods. As expected, automatic temporal preparation (sequential effects) was not affected by our circadian manipulation. However, we did 
not find clear evidence supporting that controlled temporal preparation (validity effects in the trialby-trial manipulation of temporal cues of Experiment 2) depends on circadian factors.

The two experiments further converged in replicating the circadian pattern of body temperature. It is known that armpit temperature shows circadian rhythmicity that correlates positively with core body temperature, such that it increases during the evening and then falls along the night reaching a minimum, coinciding with the lowest level in vigilance (Baehr et al., 2000). Our finding of higher temperature in the evening than in the morning session confirmed that participants performed the task in two different phases of the circadian cycle. Although we did not expect this time of day effect to be similar for both chronotypes, the result has been reported in other studies (Horne, Brass, \& Pettitt, 1980; West et al., 2002). It is likely that more frequent sampling of temperature along the day would maximise the expected differences.

The subjective measure of activation state, on the other hand, was sensitive to the synchrony effect, especially in Experiment 2, where time of day produced opposite effects on subjective activation for morning-type vs. evening-type groups (Monk, 1989). This effect appeared selective to alertness rather than emotion, as reports of subjective affect were virtually unaffected in this research. The fact that participants felt more active at their optimal time of day according to their chronotype was also reflected in objective measurements, like the overall RT in the temporal preparation task of both experiments. This finding suggests that participants had optimal vigilance at their optimal time of day, which may involve more arousal, more cognitive resources and less mental fatigue to perform the RT task with increased efficiency (Kahneman, 1973; Langner et al., 2010; Robertson \& O’Connell, 2010; Yerkes \& Dodson, 1908). This increased efficiency, surprisingly, did not influence temporal preparation. 
According to our hypothesis, we expected to find a synchrony effect selectively on temporal preparation processes related to executive control, that is, on temporal orienting rather than sequential effects. This prediction was not confirmed by the current research. However, the lack of synchrony effects on temporal orienting does not necessarily question the role of executive control in this process of temporal preparation. In fact, research has shown temporal orienting to be impaired when executive control is concurrently demanded by a working memory task in a dualtask procedure (Capizzi, et al., 2013); temporal orienting is also altered in populations typically associated to dysfunctional prefrontal cortex, such as fibromyalgia and frontal patients (Correa et al., 2011; Triviño et al., 2010). Therefore, it may be possible that our behavioural measures were not sensitive to putative synchrony effects on controlled processes underlying temporal orienting. In this vein, a recent fMRI study (Schmidt et al., 2012) measured the effects of chronotype and time of day on the performance of a stroop task and found that the synchrony effect was only apparent on brain activity related to executive control, but not on behavioural performance measures. Likewise, it is possible to speculate that, in our study, neural activity related to controlled temporal preparation (Coull \& Nobre, 1998) could have been modulated by our circadian manipulation without clear expression at the behavioural level. This intriguing issue should be addressed by future studies including measures of brain activity.

Other potential explanations regarding the lack of synchrony effects on temporal preparation may consider that a demanding attentional task can influence the circadian state of participants (Gritton, Sutton, Martinez, Sarter, \& Lee, 2009). That is, the arousing component of a demanding task itself could have compensated the reduced level of vigilance when participants performed at non-optimal times of day. This masking effect may be exacerbated by the use of warning signals in our task, which probably increased alertness. Thus, an interesting line of research will be to study the interactions between tonic and phasic alertness. It might also be argued that the synchrony effect 
was not strong enough in our experiments. For example, the evening session (9 pm) was probably not excessively late (considering Spanish schedules; Adan \& Almirall, 1991) for morning-type participants, which explains the lack of time of day effects in Experiment 1. It is then possible that testing at later hours could have been more effective. Another manipulation to further test circadian effects on temporal preparation in future studies could involve increasing contextual temporal uncertainty by using more than two foreperiods and of longer duration. For example, Steinborn and Langner (2012) found stronger sequential effects in experiments with high rather than low temporal uncertainty (e.g., foreperiods of 1200, 2400 and $3600 \mathrm{~ms}$ ). In line with the logic of our Experiment 2, increasing the demands of temporal preparation could optimise the finding of circadian influences. Finally, the use of constant routine and forced desynchrony protocols would probably be more sensitive to study circadian rhythmicity of temporal preparation processes (Blatter \& Cajochen, 2007).

Despite the abovementioned conditions might optimize the finding of synchrony effects on temporal preparation, it is important to note that under the current conditions the overall RT still showed to be sensitive to our main manipulation. Both power analyses and optimised analyses in the subgroup of participants showing the largest synchrony effect (Langner et al., 2010) provided further support to the resistance of temporal preparation to circadian influence. Our results therefore indicate that only processes mediating overall RT (vigilance) but not processes related to temporal preparation (executive control) were susceptible to circadian factors (see Langner et al., 2010, for similar arguments).

As interim conclusion, the current research supported the dissociation between circadian timing and temporal preparation. Our findings also add to the literature interested in the interplay between vigilance and temporal preparation. Both phenomena commonly involve the function of regulating 
the deployment of attentional resources over time; however, there are only a few studies addressing their mutual interactions, and the results are inconclusive yet. For example, Langner and colleagues (2010) studied the effects on temporal preparation produced by "mental fatigue" from prolonged time on task. They used a variable foreperiod paradigm to measure both foreperiod and sequential effects. Participants performed a simple RT task during 50 minutes. The authors assumed that increasing mental fatigue along the task would selectively impair controlled but not automatic processes of temporal preparation. Since the results showed that mental fatigue, as indexed by an overall RT slowing along time on task, did not influence either foreperiod or sequential effects, it was concluded that these two effects were mediated by automatic rather than controlled processing. In contrast, we have recently found that sequential effects can change as a function of arousal, by manipulating the time interval allowed for resting between two consecutive trials (Vallesi, Lozano, \& Correa, 2013). These and our current findings invite to further investigations on the relationship between arousal and temporal preparation, for example, by taking more direct measures of arousal, such as the EEG. 


\section{Acknowledgments}

This work was supported by the Spanish Ministerio de Ciencia e Innovación to A.C. (Ramón y Cajal programme: RYC-2007-00296 and PLAN NACIONAL de I+D+i: PSI2010-15399). The authors are grateful to Beatriz Blanca for her collaboration during data collection. 


\section{References}

Adan, A., \& Almirall, H. (1991). Horne and Östberg Morningness--eveningness questionnaire: A reduced scale. Personality and Individual Differences, 12, 241-253.

Adan, A., Archer, S. N., Hidalgo, M. P., Di Milia, L., Natale, V., \& Randler, C. (2012). Circadian Typology: A Comprehensive Review. Chronobiology International, 29(9), 1153-1175. doi:10.3109/07420528.2012.719971

Agostino, P. V., Golombek, D. A., \& Meck, W. H. (2011). Unwinding the molecular basis of interval and circadian timing. Frontiers in integrative neuroscience, 5, 64.

doi:10.3389/fnint.2011.00064

Baehr, E. K., Revelle, W., \& Eastman, C. I. (2000). Individual differences in the phase and amplitude of the human circadian temperature rhythm: with an emphasis on morningnesseveningness. Journal of Sleep Research, 9(2), 117-127.

Berendes, H., Marte, E., Ertel, R., McCarthy, J., Anderson, J., \& Halberg, F. (1960). Circadian physiologic rhythms and lowered blood 5- hydroxytryptamine in human subjects with defective mentality. Physiologist, (3), 20.

Blatter, K., \& Cajochen, C. (2007). Circadian rhythms in cognitive performance: methodological constraints, protocols, theoretical underpinnings. Physiology \& Behavior, 90(2-3), 196-208. doi:10.1016/j.physbeh.2006.09.009

Buhusi, C. V., \& Meck, W. H. (2005). What makes us tick? Functional and neural mechanisms of interval timing. Nature Reviews Neuroscience, 6, 755-765.

Capizzi, M., Correa, A., \& Sanabria, D. (2013). Temporal orienting of attention is interfered by concurrent working memory updating. Neuropsychologia. doi:10.1016/j.neuropsychologia.2012.10.005

Capizzi, M., Sanabria, D., \& Correa, A. (2012). Dissociating controlled from automatic processing 
in temporal preparation. Cognition, 123, 293-302. doi:10.1016/j.cognition.2012.02.005

Cohen, J. (1983). The Cost of Dichotomization. Applied Psychological Measurement, 7(3), 249253. doi:10.1177/014662168300700301

Correa, A. (2010). Enhancing behavioural performance by visual temporal orienting. In A. C. Nobre \& J. T. Coull (eds.), Attention and Time (pp. 357-370). Oxford: Oxford University Press.

Correa, A., Cappucci, P., Nobre, A. C., \& Lupiáñez, J. (2010). The two sides of temporal orienting: Facilitating perceptual selection, disrupting response selection. Experimental Psychology, $57(2), 142-148$.

Correa, A., Lupiáñez, J., Milliken, B., \& Tudela, P. (2004). Endogenous temporal orienting of attention in detection and discrimination tasks. Perception and Psychophysics, 66(2), 264278.

Correa, A., Miró, E., Martínez, M. P., Sánchez, A. I., \& Lupiáñez, J. (2011). Temporal preparation and inhibitory deficit in fibromyalgia syndrome. Brain and Cognition, 75(3), 211-216.

Correa, A., Sanabria, D., Spence, C., Tudela, P., \& Lupiáñez, J. (2006). Selective temporal attention enhances the temporal resolution of visual perception: Evidence from a temporal order judgment task. Brain Research, 1070(1), 202-205.

Correa, A., Triviño, M., Pérez-Dueñas, C., Acosta, A., \& Lupiáñez, J. (2010). Temporal preparation, response inhibition and impulsivity. Brain and Cognition, 73(3), 222-228.

Coull, J. T., Davranche, K., Nazarian, B., \& Vidal, F. (2012). Functional anatomy of timing differs for production versus prediction of time intervals. Neuropsychologia. doi:10.1016/j.neuropsychologia.2012.08.017

Coull, J. T., Frith, C. D., Buchel, C., \& Nobre, A. C. (2000). Orienting attention in time: Behavioural and neuroanatomical distinction between exogenous and endogenous shifts. Neuropsychologia, 38(6), 808-819.

Coull, J. T., \& Nobre, A. C. (1998). Where and when to pay attention: The neural systems for 
directing attention to spatial locations and to time intervals as revealed by both PET and fMRI. The Journal of Neuroscience, 18(18), 7426-7435.

Coull, J. T., \& Nobre, A. C. (2008). Dissociating explicit timing from temporal expectation with fMRI. Current Opinion in Neurobiology, 18, 1-8.

García, A., Ramírez, C., Martínez, B., \& Valdez, P. (2012). Circadian rhythms in two components of executive functions: cognitive inhibition and flexibility. Biological Rhythm Research, 43(1), 49-63. doi:10.1080/09291016.2011.638137

Gritton, H. J., Sutton, B. C., Martinez, V., Sarter, M., \& Lee, T. M. (2009). Interactions between cognition and circadian rhythms: attentional demands modify circadian entrainment. Behavioral Neuroscience, 123(5), 937-948. doi:10.1037/a0017128

Horne, J. A., Brass, C. G., \& Pettitt, A. N. (1980). Ciradian performance differences between morning and evening «types». Ergonomics, 23(1), 29-36. doi:10.1080/00140138008924715

Horne, J. A., \& Ostberg, O. (1976). A self-assessment questionnaire to determine morningnesseveningness in human circadian rhythms. International Journal of Chronobiology, 4(2), 97110.

Kahneman, D. (1973). Attention and effort. London: Prentice Hall.

Katzenberg, D., Young, T., Finn, L., Lin, L., King, D. P., Takahashi, J. S., \& Mignot, E. (1998). A CLOCK polymorphism associated with human diurnal preference. Sleep, 21(6), 569-576.

Kleitman, N. (1933). Studies on the physiology of sleep: VIII. Diurnal Variation in Performance. American Journal of Physiology -- Legacy Content, 104(2), 449 -456.

Langner, R., Steinborn, M. B., Chatterjee, A., Sturm, W., \& Willmes, K. (2010). Mental fatigue and temporal preparation in simple reaction-time performance. Acta Psychologica, 133(1), 6472. doi:10.1016/j.actpsy.2009.10.001

Lim, J., \& Dinges, D. F. (2008). Sleep deprivation and vigilant attention. Annals of the New York Academy of Sciences, 1129, 305-322. doi:10.1196/annals.1417.002 
Los, S. A., \& Van den Heuvel, C. E. (2001). Intentional and unintentional contributions to nonspecific preparation during reaction time foreperiods. Journal of Experimental Psychology: Human Perception and Performance, 27, 370-386.

Manly, T., Lewis, G. H., Robertson, I. H., Watson, P. C., \& Datta, A. K. (2002). Coffee in the cornflakes: time-of-day as a modulator of executive response control. Neuropsychologia, 40(1), 1-6.

Matchock, R. L., \& Mordkoff, J. T. (2009). Chronotype and time-of-day influences on the alerting, orienting, and executive components of attention. Experimental Brain Research, 192(2), 189-98. doi:10.1007/s00221-008-1567-6

May, C. P., \& Hasher, L. (1998). Synchrony effects in inhibitory control over thought and action. Journal of Experimental Psychology. Human Perception and Performance, 24(2), 363-379.

May, C. P., Hasher, L., \& Foong, N. (2005). Implicit memory, age, and time of day: paradoxical priming effects. Psychological Science, 16(2), 96-100.

Monk, T. H. (1989). A Visual Analogue Scale technique to measure global vigor and affect. Psychiatry research, 27(1), 89-99.

Niemi, P., \& Näätänen, R. (1981). Foreperiod and simple reaction time. Psychological Bulletin, 89, 133-162.

Nobre, A. C. (2001). Orienting attention to instants in time. Neuropsychologia, 39, 1317-1328.

Nobre, A. C., Correa, A., \& Coull, J. T. (2007). The hazards of time. Current Opinion in Neurobiology, 17, 1-6.

Ortiz-Tudela, E., Martinez-Nicolas, A., Campos, M., Rol, M. Á., \& Madrid, J. A. (2010). A New Integrated Variable Based on Thermometry, Actimetry and Body Position (TAP) to Evaluate Circadian System Status in Humans. PLoS Comput Biol, 6(11), e1000996. doi:10.1371/journal.pcbi.1000996 
Posner, M. I. (1978). Chronometric explorations of mind. Hillsdale, NJ: Lawrence Erlbaum Associates.

Robertson, I. H., \& O’Connell, R. (2010). Vigilant attention. In Attention and Time.

Ryan, L., Hatfield, C., \& Hofstetter, M. (2002). Caffeine reduces time-of-day effects on memory performance in older adults. Psychological science, 13(1), 68-71.

Sarabia, J. A., Rol, M. A., Mendiola, P., \& Madrid, J. A. (2008). Circadian rhythm of wrist temperature in normal-living subjects A candidate of new index of the circadian system. Physiology \& behavior, 95(4), 570-580. doi:10.1016/j.physbeh.2008.08.005

Schmidt, C., Peigneux, P., Leclercq, Y., Sterpenich, V., Vandewalle, G., Phillips, C., ... Collette, F. (2012). Circadian Preference Modulates the Neural Substrate of Conflict Processing across the Day. PloS One, 7(1), e29658. doi:10.1371/journal.pone.0029658

Schneider, W., Eschman, A., \& Zuccolotto, A. (2002). E-Prime user's guide. Pittsburgh: Psychology Software Tools Inc.

Steiger, J., \& Fouladi, R. (1997). Noncentrality interval estimation and the evaluation of statistical models. In L. Harlow, S. Mulaik, \& J. Steiger (eds.), What if there were no significance tests. New Jersey: Lawrence Erlbaum.

Steinborn, M. B., \& Langner, R. (2011). Distraction by irrelevant sound during foreperiods selectively impairs temporal preparation. Acta psychologica, 136(3), 405-418. doi:10.1016/j.actpsy.2011.01.008

Steinborn, M. B., \& Langner, R. (2012). Arousal modulates temporal preparation under increased time uncertainty: Evidence from higher-order sequential foreperiod effects. Acta Psychologica, 139(1), 65-76. doi:10.1016/j.actpsy.2011.10.010

Triviño, M., Arnedo, M., Lupiáñez, J., Chirivella, J., \& Correa, A. (2011). Rhythms can overcome temporal orienting deficit after right frontal damage. Neuropsychologia, 49, 3917-30. doi:10.1016/j.neuropsychologia.2011.10.009 
Triviño, M., Correa, A., Arnedo, M., \& Lupiáñez, J. (2010). Temporal orienting deficit after prefrontal damage. Brain, 133, 1173-1185.

Valdez, P., Ramírez, C., García, A., Talamantes, J., \& Cortez, J. (2010). Circadian and homeostatic variation in sustained attention. Chronobiology international, 27(2), 393-416. doi:10.3109/07420521003765861

Vallesi, A., Lozano, V., \& Correa, A. (2013). Dissociating temporal preparation processes as a function of the inter-trial interval duration. Cognition, 127, 22-30.

Vallesi, A., Mussoni, A., Mondani, M., Budai, R., Skrap, M., \& Shallice, T. (2007a). The neural basis of temporal preparation: insights from brain tumor patients. Neuropsychologia, 45(12), 2755-2763.

Vallesi, A., \& Shallice, T. (2007). Developmental dissociations of preparation over time: deconstructing the variable foreperiod phenomena. Journal of Experimental Psychology: Human Perception and Performance, 33(6), 1377-1388.

Vallesi, A., Shallice, T., \& Walsh, V. (2007b). Role of the prefrontal cortex in the foreperiod effect: TMS evidence for dual mechanisms in temporal preparation. Cerebral Cortex, 17(2), 46674.

West, R., Murphy, K. J., Armilio, M. L., Craik, F. I. M., \& Stuss, D. T. (2002). Effects of time of day on age differences in working memory. The Journals of Gerontology. Series B, Psychological Sciences and Social Sciences, 57(1), P3-10.

Wright, K. P., Hull, J. T., \& Czeisler, C. A. (2002). Relationship between alertness, performance, and body temperature in humans. American Journal of Physiology. Regulatory, Integrative and Comparative Physiology, 283(6), R1370-1377. doi:10.1152/ajpregu.00205.2002

Yerkes, R. M., \& Dodson, J. D. (1908). The relation of strength of stimulus to rapidity of habitformation. Journal of Comparative Neurology and Psychology, 18, 459-482. 


\section{Notes}

1. Statistics of these measures had different degrees of freedom because data from some participants were missing or could not be registered for technical reasons.

2. As suggested by a reviewer, we also tested whether variability of responding (SD) showed a synchrony effect. Standard deviations of RTs per condition and participant were submitted to chronotype $\mathrm{x}$ time of day $\mathrm{x}$ validity $\mathrm{x}$ foreperiod ANOVAs. The results replicated the same pattern as with mean RTs, that is, there was a significant synchrony effect in overall SD of RT for both Experiment $1, F(1,23)=7.96, p=.01$, and Experiment $2, F(1,28)=6.80, p=.01$, but no significant effects concerning our variables of interest, validity and previous foreperiod. 